\title{
Robust Assessment of Shear Parameters from direct shear tests
}

\section{Wolfgang Fellin}

Geotechnical and Tunnel Engineering, University of Innsbruck, A-6020 Innsbruck, Austria

E-mail: wolfgang.fellin@uibk.ac.at

\section{Michael Oberguggenberger*}

Engineering Mathematics, University of Innsbruck, A-6020 Innsbruck, Austria

E-mail: michael.oberguggenberger@uibk.ac.at

*Corresponding author

\begin{abstract}
In the geotechnical determination of the cohesion $c$ and the angle of internal friction $\varphi$ of a soil from shear tests, a linear regression model is fitted to normal and shear stress data, and confidence bounds are computed. The applicability of standard linear regression is limited by the physical requirement of nonnegative cohesion and the statistical requirement of normality. We propose two methods from computational statistics that are able to overcome both obstacles: a bootstrap resampling method in case the experimental data set is sufficiently large, and a Bayesian approach for small samples. The methods are demonstrated at the hand of a real data set for glacial silt.
\end{abstract}

Keywords: Shear parameters; non-normal regression; Bayesian methods; bootstrap confidence intervals; computational statistics.

Reference to this postprint-version should be made as follows: Fellin, W. and Oberguggenberger, M. (2012) 'Robust Assessment of Shear Parameters from direct shear tests', Int. J. Reliability and Safety, Vol. 6, No. 1/2/3, pp.49-64. doi: 10.1504/IJRS.2012.044294, https:

//www . inderscience. com/info/inarticle.php?artid=44294

Biographical notes: Wolfgang Fellin received the Ph.D. degree in civil engineering from the University of Innsbruck, Austria, in 1999. From 1995 to 2003, he was an Assistant Professor with the Institute of Geotechnical and Tunnelling Engineering, University of Innsbruck. Since 2003, he has been an Associate Professor with the Institute of Infrastructure, University of Innsbruck. He is member of the committee for laboratory experiments of the German national codes. His research interests are deep vibration compaction, constitutive modelling, time integration of constitutive models, snow and ice mechanics, and reliability theory.

Copyright (c) 2012 Inderscience Enterprises Ltd. 
Michael Oberguggenberger received a Master's degree in Mathematics and Physics from the University of Innsbruck in Austria in 1979 and a PhD in Mathematics from Duke University in Durham, North Carolina in 1981. Since 1982 he has been employed at the Faculty of Civil Engineering of the University of Innsbruck, Austria. He is Full Professor and Head of the Unit of Engineering Mathematics there. He is vice-president of the Austrian Mathematical Society. His research expertise includes partial differential equations, mathematical physics, stochastic analysis, operations research, engineering mathematics, and risk analysis.

\section{Introduction}

Civil engineering design includes the consideration of ultimate limit states and of serviceability limit states. These limit states can formally be written by means of a limit state function $g(F, X)$, where $F$ are the actions (e.g. forces) and $X$ the parameters of the involved materials (e.g. shear strength). Negative values of $g(F, X)$ correspond to failure in the case of ultimate limit state. Highly sophisticated geotechnical design can be performed using a probabilistic concept. Modelling $X$ and $F$ as random variables, one can compute an operational failure probability

$$
p_{f}=P(g(F, X)<0)
$$

provided the probability distributions of $F$ and $X$ and their distribution parameters are known.

However, the current Eurocodes employ the so-called semi-probabilistic design. For each design situation one has to define the resistance of the structure $R$ and the effect of the actions $E$. The limit state function writes then $g=R-E$. In geotechnical design both quantities may be nonlinear functions of the actions and the material parameters: $R(F, X), E(F, X)$. The variability of the variables $X$ and $F$ is represented by using deterministic characteristic values $F_{k}$ and $X_{k}$ and partial safety factors (to reduce favourable quantities and enlarge unfavourable ones) to finally arrive at the design values of the effects $E_{d}$ and the resistance $R_{d}$. The designing engineer has to verify a relation of the type

$$
E_{d} \leq R_{d} .
$$

The partial safety factors and the way how they are applied to actions and material parameters, effects of actions and resistance or other combinations are prescribed in the codes. One way is simple to apply them directly to $R$ and $E$

$$
E_{d}=\gamma_{E} E\left(F_{k}, X_{k}\right) \leq R\left(F_{k}, X_{k}\right) / \gamma_{R}=R_{d} .
$$

When using these codes, one of the principal tasks in geotechnical engineering is the selection of characteristic values of soil parameters. Statistical methods can serve as a rational guideline through this difficult task and may reduce the influence of some subjective judgements. 


\subsection{Characteristic values of a soil property}

Results from field or laboratory tests are used to asses the parameters governing the model of interest. These parameters are denoted as measured values. Theoretical or empirical relationships or correlations result in so-called derived values, see Orr (2000). For example, the torque measured in a field vain test is correlated to the undrained cohesion $c_{u}$ by means of an empirical relation. The characteristic value should be a cautious estimate of the derived value based on the design situation, the structure and the volume of ground involved. If local failure can be excluded in the situation under scrutiny, the characteristic value is a cautious estimate of the mean value of the soil parameter affecting the occurrence of a limit state.

For uncorrelated parameters this can easily be worked out with classical statistics with the help of a two sided $90 \%$ confidence interval obtained from the student-t distribution, see e.g. Orr (2000); Bauduin (2002). The confidence interval tends to be broad if only few experiments are available. Based on comparative calculations, setting the width of the confidence interval to the standard deviation was proposed in such cases by Schneider (1999). Another simplified approach is to apply a correction factor to the derived value, which depends on the coefficient of variation; Watabe et al. (2009). If a soil property can be assumed to have a linear trend with increasing depth, the characteristic value in a certain depth can be estimated by means of the confidence region of a linear regression; Bauduin (2002). The simplified correction factor approach can also be used in such cases; Watabe et al. (2009). A Bayesian approach is used to incorporate comparable experience, see e.g. Orr (2000).

Although cohesion and friction angle of a soil are often assumed to be uncorrelated, it is clear that they are actually correlated, simply because they are parameters of a linear regression. A linearisation of the confidence region of the Coulomb shear strength line was proposed in order to tackle this issue by Kruse (2003), however, without presenting the specific calculation method. Linear outer boundaries of the confidence region in a specific stress range are proposed to achieve a minimal admissible stress region in Fellin et al. (2009).

The present paper is based on this approach, which will be further developed and combined with more advanced probabilistic concepts. We begin by outlining the linearisation of the shear strength.

\subsection{Characteristic shear parameters}

The shear strength $\tau_{f}$ is expressed by the cohesion $c$ and the angle of internal friction $\varphi$. Eurocode 7 requires that laboratory and in situ experiments have to be supplemented by experience in comparable situations and, in case statistical procedures are used, $5 \%$ and $95 \%$ confidence limits have to be observed for the characteristic values; Eurocode 7 (2004).

The shear strength of soil can be displayed in a stress diagram, where the shear stress $\tau$ is plotted versus the effective normal stress $\sigma$ (Figure 1-left). The shear strength is a generally curved line separating stress states which can be sustained by the soil (gray region in Figure 1) from stress states that cannot appear. Stress 
states on the line will cause local failure. A linearisation in a specific stress range is usually used for design by means of Coulomb's model

$$
\tau_{f}=c+\sigma \tan \varphi,
$$

with the cohesion $c$ and the angle of internal friction $\varphi$ (Figure 1-right). The stress range has to be chosen properly by the designing engineer according to the expected stress state in the projected geotechnical construction.
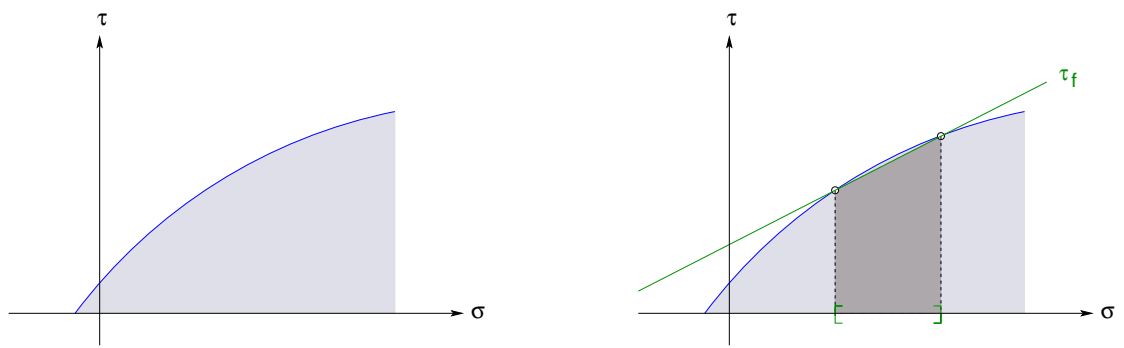

Figure 1 Shear strength of soil (left); linearised in a specific stress range (right).

The shear strength $\tau_{f}$ can be determined by direct shear tests for example. A linear regression with the resulting pairs of normal stress and shear stress at failure of the sample yield the mean values of cohesion and friction angle. The confidence region for this estimated shear strength line can be calculated by standard statistical procedures (Figure 2-left). In civil engineering a $90 \%$ confidence level is of interest, since the probability of the shear strength being less than the lower limit of the region is only $5 \%$, which is in agreement with the requirements of Eurocode 7 .
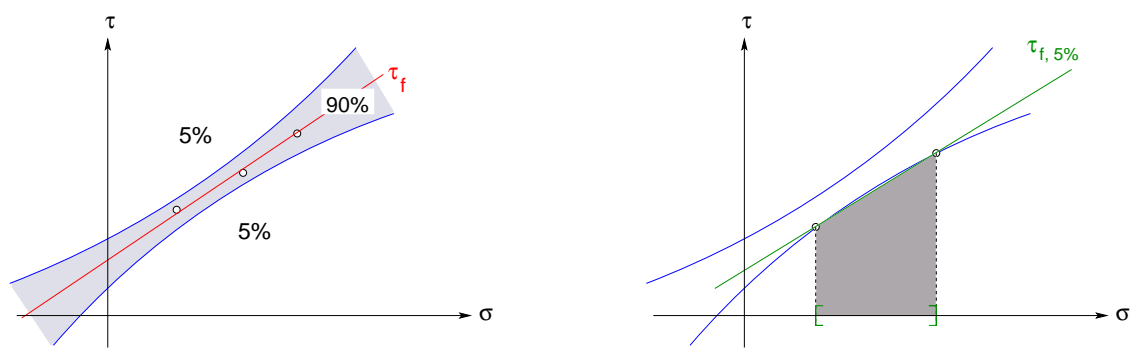

Figure 2 90\% confidence range of regression (left); linearised confidence limit (right).

A further linearisation of the lower confidence limit in a specific stress range (Figure 2-right) gives the stress line $\tau_{f, 5 \%}$, which is given by the equation

$$
\tau=c_{5 \%}+\sigma \tan \varphi_{5 \%}
$$

in the $(\sigma, \tau)$-plane. The stress line $\tau_{f, 5 \%}$ can serve for assessing the characteristic shear strength, see Fellin et al. (2009). We call the pair $\left(c_{5 \%}, \varphi_{5 \%}\right)$ the $5 \%$-limit of the parameters $c$ and $\varphi$. A linearisation of the upper confidence limit yields $\left(c_{95 \%}\right.$, $\left.\varphi_{95 \%}\right)$. 


\subsection{Mathematical aspects}

There are two obstacles for the standard linear regression model: First, in cases where the mean value of the cohesion is close to zero, the standard approach may result in negative $c_{5 \%}$ values. Second, the regressor variables and hence the regression coefficients may fail to be normally distributed.

In principle, the first problem can be dealt with by replacing the quadratic optimisation problem in the regression analysis by a quadratic optimisation with constraints. This leads to subcases of regression with or without intercepts as well as to definitely not normally distributed variables. In this paper, we propose two approaches to address non-normality and to obtain geotechnically acceptable confidence limits.

In the case of availability of a sufficiently large sample size $(\geq 15)$, we propose a computational approach that provides bootstrap confidence intervals by repeated resampling of the data. This approach does not require normality and the $c \geq 0$ constraint can be naturally guaranteed.

In the case of small sample sizes $(<5)$ we find a Bayesian approach appropriate that replaces the confidence intervals by high probability density regions of the posterior distribution. Here we face the problem of assigning the prior distribution. We show how noninformative priors (Martz et al., 1982, Section 6.1) can be plausibly chosen as uniform distributions on suitable intervals. We briefly indicate how the method could be extended to families of priors, that is, a robust Bayesian approach.

In both approaches, current methods from computational statistics have to be employed, resulting in credible confidence limits for the characteristic values.

For clarity, we point out an issue of model calibration. The values actually to be assessed are the expectation values $\mathrm{E}(c)$ and $\mathrm{E}(\varphi)$ of the cohesion and the angle of internal friction, respectively. Both the bootstrap resampling technique and the Bayesian approach aim at assessing the probability distribution of the expectation values. The confidence intervals computed by these methods thus are estimates of the variability of the mean of $c$ and the mean of $\varphi$. As outlined in the Introduction, the confidence limits constitute the information required in engineering design for determining the characteristic values. That is why we do not elaborate on further statistical parameters that could be extracted from our procedures (except for the correlation between $c$ and $\varphi$, which is assessed implicitly when calibrating our models). In the Bayesian approach, the counterpart of confidence regions are so-called highest posterior density regions, cf. (Martz et al., 1982, Section 5.5.4), (Viertl, 2003, Section 40.3). Accordingly, these regions form our concern when employing Bayesian methods in order to assess the required confidence limits.

\section{General idea of proposed methods}

As outlined above, standard regression gives an estimate of the $90 \%$ confidence range as depicted in Figure 2-left. This estimate is based on the assumption of normality. In this geotechnical problem, the assumption is questionable and definitely violated if the $c \geq 0$ constraint is imposed. In order to arrive at an estimate of a specific stress range as in Figure 2-right, we need to know the joint 
distribution of $c$ and $\tan \varphi$, or at least a bivariate sample $\left(c_{i}, \tan \varphi_{i}\right), i=1, \ldots, M$, thereof. Each data pair $\left(c_{i}, \tan \varphi_{i}\right)$ determines a shear stress line; our task will be to determine the population of lines that lie within a $90 \%$ confidence range. The actual limiting stress line $\tau_{f, 5 \%}$ will be inferred by a geometrical enveloping procedure.

\subsection{Determination of $90 \%$ confidence ranges}

In the sequel, we will produce samples $\left(c_{i}, \tan \varphi_{i}\right), i=1, \ldots, M$, following the joint distribution of $c$ and $\tan \varphi$ by two methods: by a Bayesian approach and by bootstrap resampling. In the Bayesian approach, the $90 \%$ confidence range will be obtained as a high probability density region. In the bootstrap resampling approach, we apply and compare two different methods. The first one is probabilistic: a bivariate normal distribution is fitted to the sample $\left(c_{i}, \tan \varphi_{i}\right)$ by the method of moments, using the first and second empirical moments of the bootstrap sample. Shear and normal stress pairs are selected by whether they lie inside or outside the $90 \%$ elliptic region of the bivariate normal distribution.

The normal distribution was chosen here for reasons of comparison with widespread practice in regression analysis. Of course, other types of distributions could be used as well. In any case, the first method requires the introduction of one or the other parametric assumption. That is why we prefer the second method, which is also more adapted to an engineering point of view. We want to determine shear strength parameters such that the probability of a smaller shear strength is less than 5\%. First we must define what is a smaller shear strength. Consider the two possible shear strength lines in Figure 3. Remember that $\tau_{f}$ is considered as linearisation of the generally curved shear strength line in a specific stress range. The areas below each line in this stress range are the stress states that can be sustained by the soil. The area below line B is smaller. We state therefore that the parameters $c$ and $\varphi$ of line B are related to a smaller shear strength than those of line $\mathrm{A}$ for the given stress range.

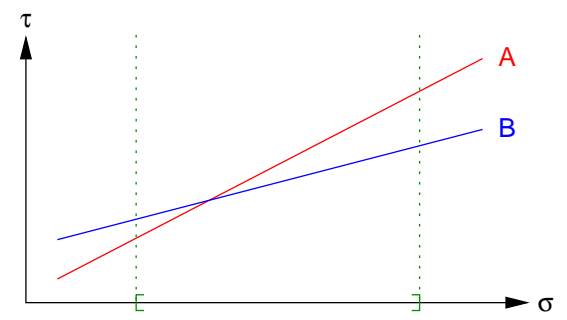

Figure 3 Two possible shear strength lines for which line B is considered to predict the smaller shear strength in the given stress strange.

We sort all parameter pairs of the possible shear strength lines with respect to the area below these lines in the given stress range. If we then exclude the upper and lower $5 \%$ of the sorted lines we end up with a fan of lines that occur with the desired estimated probability of $90 \%$. 
For brevity, we shall refer to the approach using the fitted bivariate normal distribution for determining the $90 \%$ confidence region as standard method and to the approach using the comparison of the areas as area method.

In this context, the area method is based on the geotechnical observation that a confidence limit of the shear strength should bound the region of admissible stress states acting in the soil. Recently, a general information-theoretic justification of the area method for producing confidence regions for arbitrary functions was given in Kreinovich et al. (2010).

\subsection{Definition of limit values}

Consider now the remaining fan of shear strength lines with a probability of $90 \%$, displayed as gray lines in Figure 4. The two intersection points of the lowest shear strength lines with the limits of the stress range gives what we define to be $\tau_{f, 5 \%}$. The same procedure with the upper lines gives $\tau_{f, 95 \%}$. This definition replaces the linearisation of the hyperbolas bounding the confidence region in Figure 2 obtained from classical linear regression.

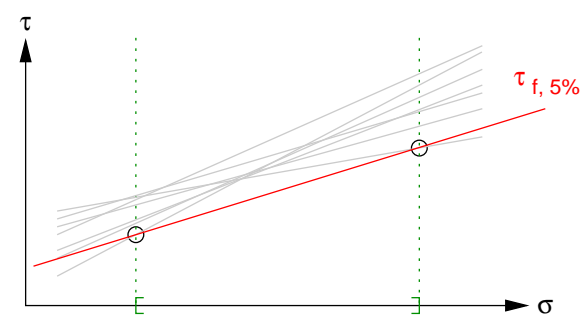

Figure 4 Definition of $\tau_{f, 5 \%}$ as line through the intersection points of the stress range and the lowest shear strength lines of the fan with $90 \%$ probability.

\section{Methods}

\subsection{Bootstrap resampling}

The starting point is a set of laboratory measurements of shear and normal stress pairs $\left(\sigma_{j}, \tau_{f, j}\right), j=1, \ldots, N$, of sufficiently large size, say $N \geq 15$. The cohesion $c$ and the friction coefficient $\tan \varphi$ are obtained as coefficients in a linear regression. A single set of measured data $\left(\sigma_{j}, \tau_{f, j}\right), j=1, \ldots, N$, gives rise to a single estimate of $c$ and $\tan \varphi$. It tells nothing about the joint distribution of the pair of parameters $(c, \tan \varphi)$.

A large sample of the joint distribution of $(c, \tan \varphi)$ can be produced by means of an empirical procedure called bootstrapping cases, cf. Montgomery et al. (2001). A bootstrap sample of size $N$ is obtained from the original sample $\left(\sigma_{j}, \tau_{f, j}\right)$ by drawing randomly $N$-times with replacement (thus a bootstrap sample may have duplicated values among the $\left.\left(\sigma_{j}, \tau_{f, j}\right)\right)$. This procedure is repeated $M$ times, say $M=1000$. Each time a linear regression is performed. In this way, a large sample of the desired cohesion and friction coefficients $\left(c_{i}, \tan \varphi_{i}\right), i=1, \ldots, M$ is 
obtained. It can be shown, Shao (1995), that the bootstrap distribution coincides asymptotically with the distribution of $(c, \tan \varphi)$ that is defined via regression by the empirical distribution of the original sample $\left(\sigma_{j}, \tau_{f, j}\right)$. For the choice of the size $M$ we refer to the literature; in the absence of detailed estimates, $M=1000$ is generally recommended. It should be pointed out that the bootstrap is a numerical simulation method and therefore comes with a numerical error: different bootstrap samples of size $M$ lead to different results - obeying the general Monte Carlo simulation error bound of magnitude $1 / \sqrt{M}$.

The generated pairs cohesion/friction coefficient $\left(c_{i}, \tan \varphi_{i}\right), i=1, \ldots, M$, determine a fan of stress lines, from which the $90 \%$ regions are computed as described in Section 2.

What concerns the geotechnical requirement of $c \geq 0$, we have various options. First, the regression coefficients $(c, \tan \varphi)$ are obtained by minimising the mean square error, or equivalently, the sum of squares $\mathrm{SS}_{\mathrm{E}}$ of the errors. We can adjoin the constraint $c \geq 0$ in this quadratic minimisation problem. When $c$ is estimated to be less than zero, this just means that a regression without $y$-intercept $(c=0)$ is performed instead. A second option is offered by the bootstrap method. We can simply reject all bootstrap samples yielding $c<0$. It turned out that in our examples about $10 \%$ of the bootstrap samples were rejected, leaving sufficiently many samples for the further analysis. Finally, one can do nothing and accept negative values for $c$, hoping that the $5 \%$ bound for $c$ is still nonnegative. This is generally not the case, but included in our presentation for comparison.

We remark that there is an alternative resampling method for producing the empirical distribution of $\left(c_{i}, \tan \varphi_{i}\right)$. This is the jackknife; see Shao (1995). In the jackknife, the new samples are not drawn by replacement, but rather as subsamples of smaller size $N-1, N-2, \ldots$ The method is combinatorially more expensive. For this reason we will not present the jackknife technique here (though we undertook all calculations with the jackknife method as well).

\subsection{Bayesian approach}

When the size $N$ of the original set of measured data is small, say $N<5$, the standard regression technique looses credibility and the bootstrapping becomes impossible (because it produces too many bootstrap samples with identical entries). In such a situation, a Bayesian approach is recommendable. We start with a brief introduction to Bayesian methods. For a detailed account of Bayesian data analysis we recommend Gelman et al. (2000).

Suppose a random variable $X$ is given whose statistical parameters we want to estimate. We concentrate here on the case that $X$ has a probability density function $p(x \mid \theta)$ that depends on certain statistical parameters $\theta$. For example, when $X$ is normally distributed, $\theta$ comprises the mean and the variance and $p(x \mid \theta)$ is the normal probability density function.

Prior knowledge is encoded in the probability density function $p(\theta)$ of the parameter $\theta$, the so-called prior distribution. Given a set of measurements, data $=$ $\left(x_{1}, \ldots, x_{N}\right)$, one may get an improved assessment of the statistical properties of the parameter $\theta$ through the posterior distribution $p(\theta \mid$ data $)$ according to Bayes' formula

$$
p(\theta \mid \text { data })=C p(\text { data } \mid \theta) p(\theta)
$$


where the normalising constant $C$ and the likelihood function $p($ data $\mid \theta)$ are given by

$$
\frac{1}{C}=\int p(\operatorname{data} \mid \theta) p(\theta) \mathrm{d} \theta, \quad p(\operatorname{data} \mid \theta)=\prod_{i=1}^{N} p\left(x_{i} \mid \theta\right),
$$

provided the data are given as an independent sample $\left(x_{1}, \ldots, x_{N}\right)$.

In order to explain how this is applied in regression analysis, we have to complete the linear model (4) by the random error term $\varepsilon$

$$
\tau_{f}=c+\sigma \nu+\varepsilon
$$

where we have written $\nu=\tan \varphi$ for clarity. The error term $\varepsilon$ has mean zero; we denote its variance by $s_{\varepsilon}^{2}$. The regressor variables are the stresses $\tau_{f}$ and $\sigma$ and play the role of the random variable $X$ above. The parameter $\theta$ is comprised by the regression coefficients $c, \nu=\tan \varphi$ and the standard deviation $s_{\varepsilon}$ of the error term. In the standard regression model, given $\sigma$, the regressor variable $\tau_{f}$ is assumed to be normally distributed with mean $c+\sigma \nu$ and variance $s_{\varepsilon}^{2}$, thus it has probability density function

$$
p(x \mid \theta)=p\left(\tau_{f} \mid c, \nu, s_{\varepsilon}\right)=\frac{1}{\sqrt{2 \pi} s_{\varepsilon}} \exp \left(-\left(\tau_{f}-c-\sigma \nu\right)^{2} / 2 s_{\varepsilon}^{2}\right) .
$$

Given a set of measurement data $=\left(\sigma_{j}, \tau_{f, j}\right), j=1, \ldots, N$, the likelihood function becomes

$$
p(\text { data } \mid \theta)=\left(\frac{1}{\sqrt{2 \pi} s_{\varepsilon}}\right)^{N} \exp \left(-\sum_{i=1}^{N}\left(\tau_{f, j}-c-\sigma_{j} \nu\right)^{2} / 2 s_{\varepsilon}^{2}\right) .
$$

Finally, we have to choose the prior distributions for $c, \nu$ and $s_{\varepsilon}$. For the sake of presentation, we work with noninformative priors here, that is, we enter in our calculations with coefficients $c, \nu$ and $s_{\varepsilon}$ which are uniformly distributed on certain intervals, denoted by $\left[c_{\min }, c_{\max }\right],\left[\nu_{\min }, \nu_{\max }\right]$, and similarly for $s_{\varepsilon}$. A main advantage of the noninformative assumption is that one can enforce the $c \geq 0$ constraint in a simple way. The choice of the other interval bounds will be specified in the next section; a discussion of other types of priors is in Section 3.4.

The posterior distribution $p(\theta \mid$ data $)=p\left(c, \nu, s_{\varepsilon} \mid\right.$ data $)$ is obtained using equation (6), where the normalising constant $C$ has to be determined by a threefold numerical integration with respect to $c, \nu$ and $s_{\varepsilon}$. Finally, the desired joint posterior distribution of $(c, \nu=\tan \varphi)$ is obtained by a further integration with respect to $s_{\varepsilon}$, and this is the result to be processed further. Actually, we employed a simplified version assuming that the variance $s_{\varepsilon}$ was known exactly (see below for the choice of $s_{\varepsilon}$ ). This way we could drop the $s_{\varepsilon}$-integration and arrived at the posterior distribution $p(c, \nu \mid$ data $)$ in one double integration step.

The task of producing a sample of values $\left(c_{i}, \nu_{i}\right)$ remains to be explained. The numerical integration delivered the values $p\left(c_{i}, \nu_{i} \mid\right.$ data $)$ of the posterior density on a chosen grid with coordinates $\left(c_{i}, \nu_{i}\right)$. From there we computed the level sets of $p(c, \nu \mid$ data $)$ by a search algorithm, and we calculated the respective total 
probability mass allocated in each level set. The level set containing $90 \%$ of all values $(c, \nu)$ was chosen as the $90 \%$ confidence region. The sample $\left(c_{i}, \nu_{i}\right)$ was defined as the set of grid points belonging to the $90 \%$ confidence region. The bounding stress lines $\tau_{f, 5 \%}$ and $\tau_{f, 95 \%}$ were then computed as in Section 2 .

\subsection{Estimation of variance and parameter interval limits}

Numerical experiments showed that leaving the regression variance $s_{\varepsilon}^{2}$ noninformative would introduce too much variability in the Bayes estimators. If $s_{\varepsilon}^{2}$ is known from geotechnical experience, this value should be taken. If it is not known, we propose here to estimate it from the data by means of the formula

$$
s_{\varepsilon} \approx \frac{q}{\sqrt{N}} \sqrt{\frac{\mathrm{SS}_{\mathrm{E}}}{N-2}}
$$

where $q$ is the $95 \%$-quantile of the Student distribution with $N-2$ degrees of freedom and $\mathrm{SS}_{\mathrm{E}}$ is the sum of squares of the residuals of the classical regression. $\mathrm{SS}_{\mathrm{E}} /(N-2)$ actually is the standard estimate for the regression variance. The scaling factor $q / \sqrt{N}$ was obtained empirically by numerical experiments and proved to produce plausible results in the geotechnical applications.

The decision about the prior bounds for $c$ and $\nu=\tan \varphi$, if not based on hard geotechnical knowledge about the soil in question, should take into account the following points. First, the lower bound $c_{\min }$ for $c$ should be nonnegative. Second, the intervals $\left[c_{\min }, c_{\max }\right]$ and $\left[\nu_{\min }, \nu_{\max }\right]$ should be large enough so that the $90 \%$ confidence region calculated from the posterior distribution lies inside the rectangle $\left[c_{\min }, c_{\max }\right] \times\left[\nu_{\min }, \nu_{\max }\right]$ which they form (too small intervals would lead to an artificial truncation). Finally, the intervals should be in a plausible range around the presumed mean values of $c$ and $\nu$. As there was no sufficient reason to exclude small values of $c$ and $\nu$, we always chose the intervals with zero lower bounds, that is, of the form $\left[0, c_{\max }\right]$ and $\left[0, \nu_{\max }\right]$. Excluding friction angles greater than $45^{\circ}$ led to the upper bound $\nu_{\max }=\tan \varphi_{\max }=1$. Finally, we chose $c=100(\mathrm{kPa})$ in all examples to follow in order to render them comparable, although this may not have been the most plausible choice in some cases.

The following three generic examples underline our claim that the proposed estimates for the prior distributions lead to plausible results. Generic outcomes of $N=3$ shear tests are listed in Table 1 .

Table 1 Generic shear experiments.

\begin{tabular}{cccc}
\hline$\sigma$ & $\tau$ series 1 & $\tau$ series 2 & $\tau$ series 3 \\
\hline 50 & 66.6 & 68.3 & 22.7 \\
150 & 120.7 & 116.1 & 73.4 \\
250 & 161.6 & 160.0 & 116.6 \\
\hline
\end{tabular}

We compare the results of the Bayesian approach with the results of a standard linear regression. Series 1 in Table 1 leads to a linear regression with moderately large variance, Series 2 produces a small regression variance, and Series 3 leads 
to a case where the standard lower confidence limit has a negative intercept, see Fig. 5. The shaded regions in Fig. 5 mark the $90 \%$ confidence regions resulting from the Bayesian approach. It is seen that they do not deviate much from the confidence regions obtained by standard regression. However, negative intercepts - as may happen when the cohesion is small - are now avoided, as can be seen in bottom plot of Fig. 5 .
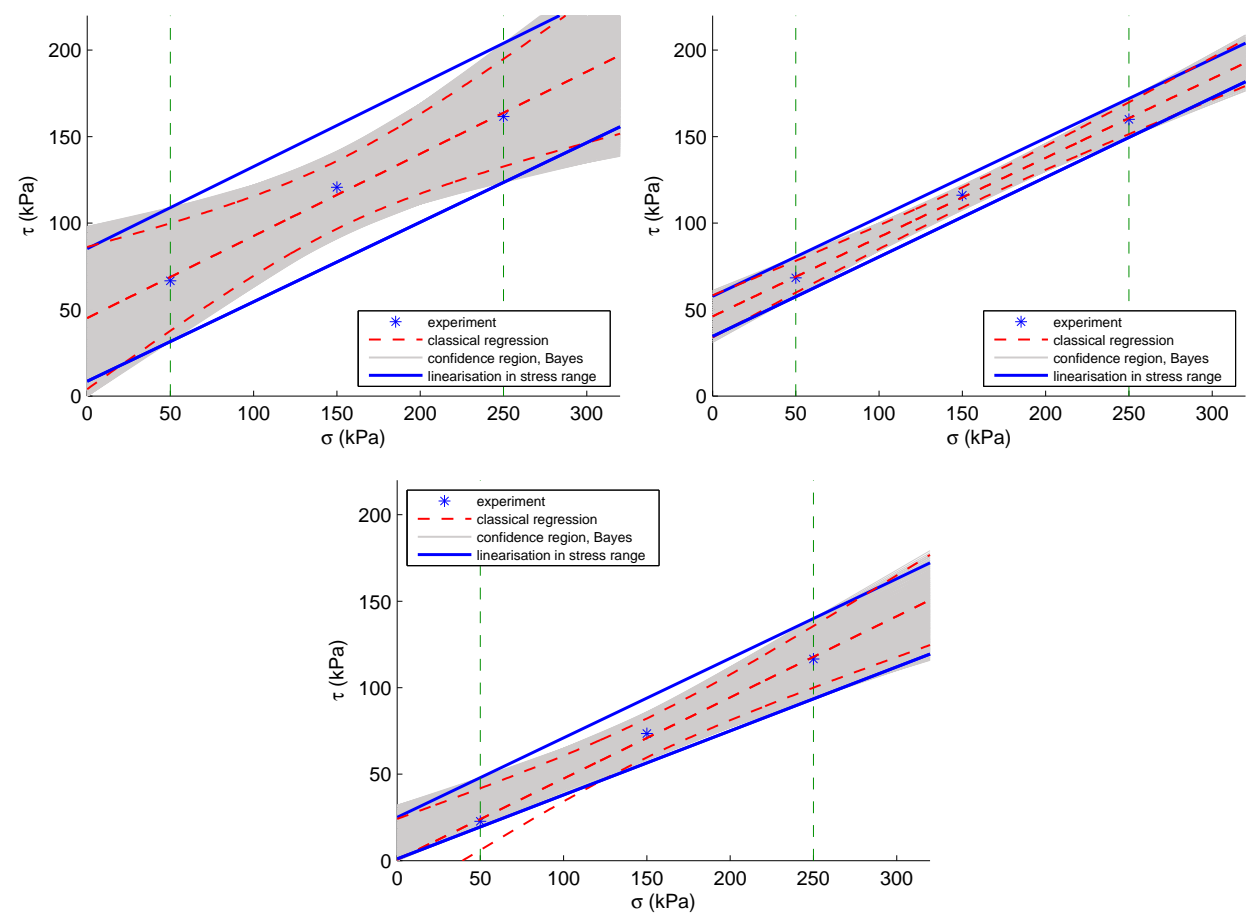

Figure 5 Generic shear test results: Bayesian approach vs. standard linear regression. The stress range used in geometrical enveloping is depicted by vertical dashed lines.

To complete the picture, we display the $90 \%$ confidence regions of the posterior distribution, approximated numerically as outlined in Section 3, referring to the generic data of Table 1 in Fig. 6. Each data point in the shaded truncated elliptic regions gives rise to a pair $(c, \nu=\tan \varphi)$ which in turn corresponds to a stress line in Fig. 5, the totality of which forms the shaded confidence regions. The lower truncations of the elliptic regions visible in Fig. 6 reflect the prior information $c \geq 0$ that was encoded in the prior distributions.

\subsection{Remarks on robust Bayesian methods}

The same procedure offers the possibility to drop the assumption of normality in the likelihood function. In fact any other family of probability density functions that can be parametrised by the mean value and the variance will do, for example, a lognormal distribution. Likewise, the prior densities need not be uniform; one 

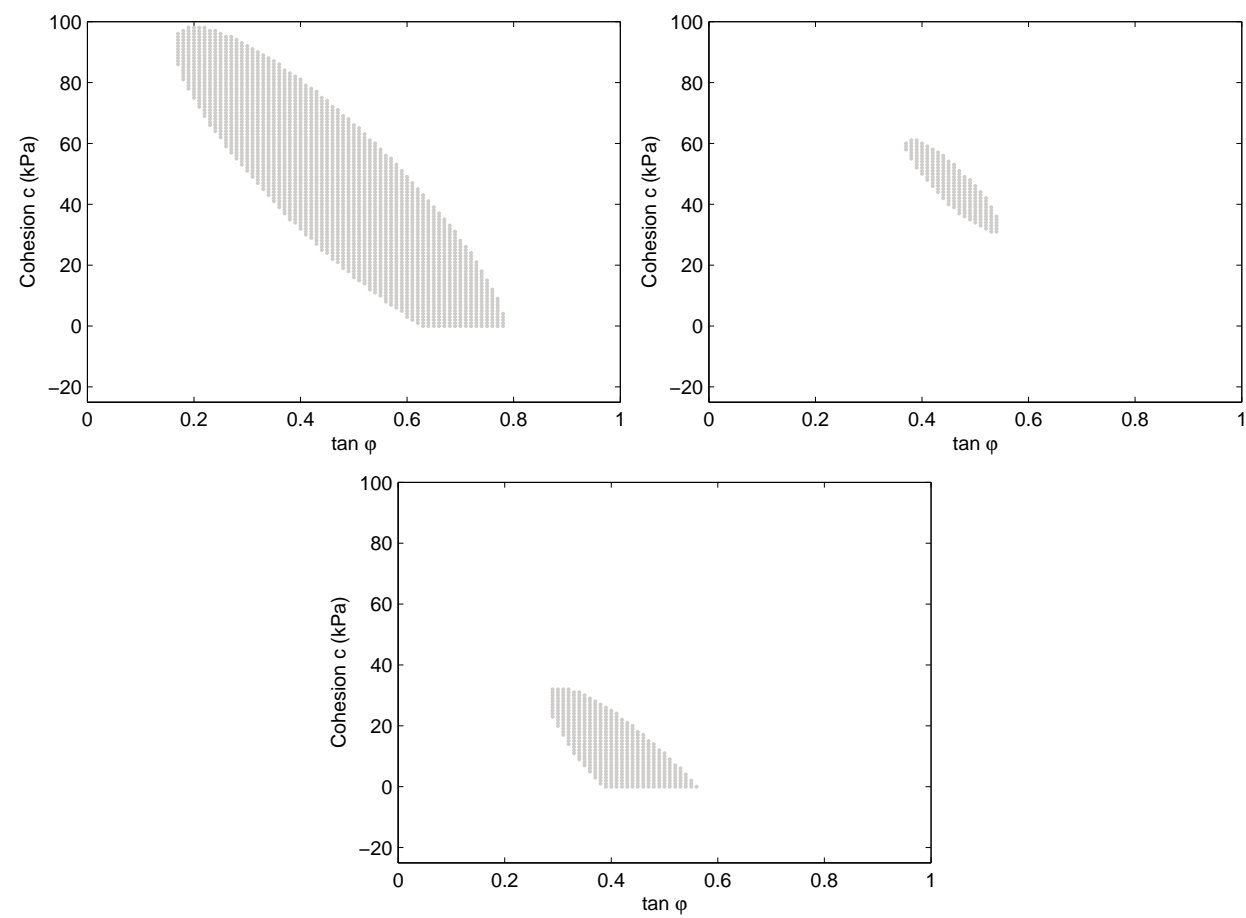

Figure $690 \%$ confidence regions (high probability density regions) of the posterior distributions.

may think of beta distributions. By repeating the same calculations leading to the $90 \%$ confidence region with different families of distributions, one may arrive at more conservative estimates. Since the methods for deriving such robust estimates are just a replication, we will not present them here.

The method is also quite insensitive with respect to the choice of the upper bounds for the noninformative priors, as long as these bounds are not too far from the respective mean values (say not more than three times the mean). Choosing very large prior intervals may lead to substantial changes in the resulting estimates for $c_{5 \%}, c_{95 \%}, \varphi_{5 \%}$ and $\varphi_{95 \%}$. Further investigations are needed to arrive at a general, geotechnically oriented proposal for the choice of the prior intervals.

\section{Application: ring shear test results}

We present the behaviour of the proposed methods on a real test series. We use the results of 14 direct ring shear tests on 4 similar samples of glacial till from Schuppener (1999), see Table 2. One engineering task may be to asses one shear parameter set for all samples, the other to deduce the shear parameters for each sample, e.g. for the first four columns in Table 2, which are the result of the first test series.

The first task can be done with bootstrapping due to the relatively large data set. The second task will be performed with the proposed Bayesian approach. 
Robust Assessment of Shear Parameters

Table 2 Results of direct ring shear tests (Schuppener, 1999, data reconstructed from Bild 1)

\begin{tabular}{l|cccc|cccccccccc}
\hline & \multicolumn{1}{|c|}{ Series 1 } & \multicolumn{10}{c}{ Series 2, 3 and 4 } \\
\hline$\sigma(\mathrm{kPa})$ & 100 & 150 & 200 & 300 & 150 & 250 & 300 & 100 & 150 & 250 & 200 & 150 & 200 & 250 \\
$\tau(\mathrm{kPa})$ & 68 & 127 & 135 & 206 & 127 & 148 & 197 & 76 & 78 & 168 & 123 & 97 & 124 & 157 \\
\hline
\end{tabular}

\subsection{Bootstrap}

The result of 1000 bootstrap realisations without any constraint is given in Fig. 7 together with the standard regression information, i.e. the regression line $(c=$ $12 \mathrm{kPa}, \varphi=31^{\circ}$ ) and the $90 \%$ confidence region. The two methods as outlined in Section 2 of estimating the $90 \%$ confidence region of the bootstrap sample are shown in the two bottom plots of Fig. 7 .
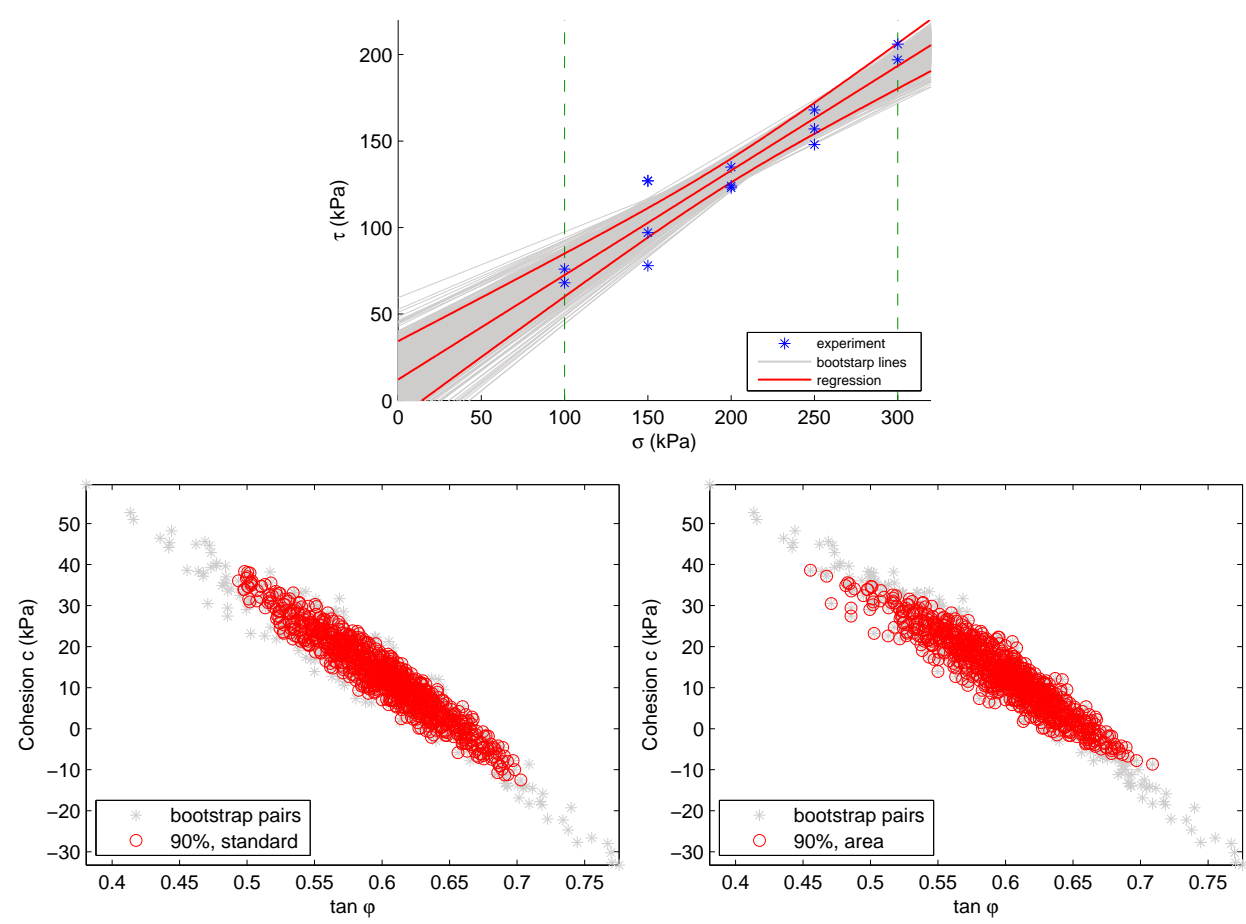

Figure 7 Bootstrap sample with 1000 realisations.

Applying the fitted bivariate normal distribution of the shear parameters as explained at the beginning of Subsection 2.1 (method standard, Fig. 7-bottom-left) or the minimum area criterion below the shear strength (method area, Fig. 7bottom-right) yield different remaining fans of shear strength lines, see Fig. 8. Both fans lie approximately within the standard confidence region, which shows that bootstrap without constraint is similar to a standard regression analysis.

Fig. 9 shows the results obtained by bootstrapping where in each linear regression the constraint $c \geq 0$ was imposed. The selection of the $90 \%$ confidence 
region was again done by method standard and method area. In this case, small differences occur that lead to the distinct linearised bootstrap confidence regions in Fig. 9.
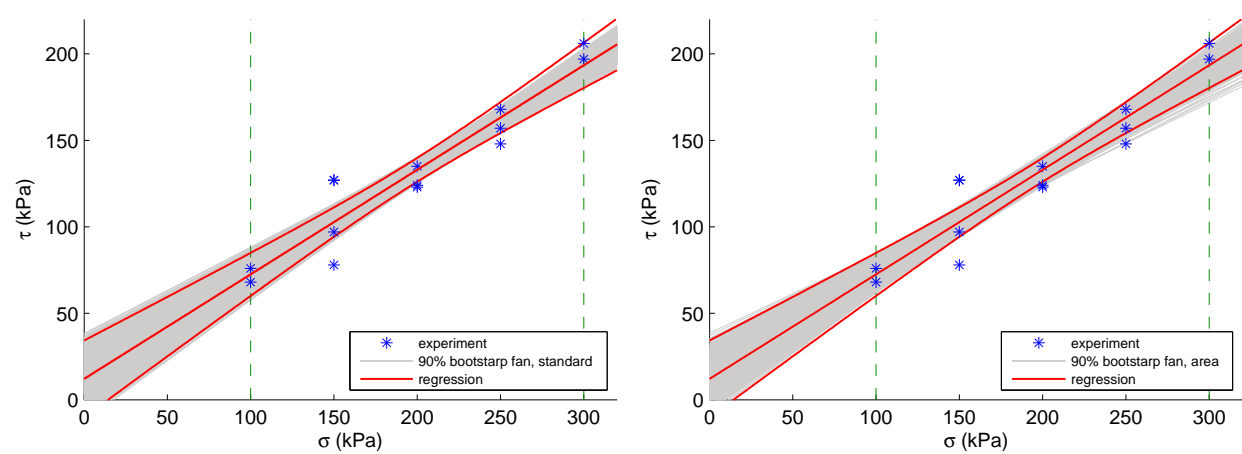

Figure 8 Bootstrap method: remaining $90 \%$ fan of realisations of Fig 7 -top, method standard vs. method area. The stress range used in method area is depicted by vertical dashed lines.
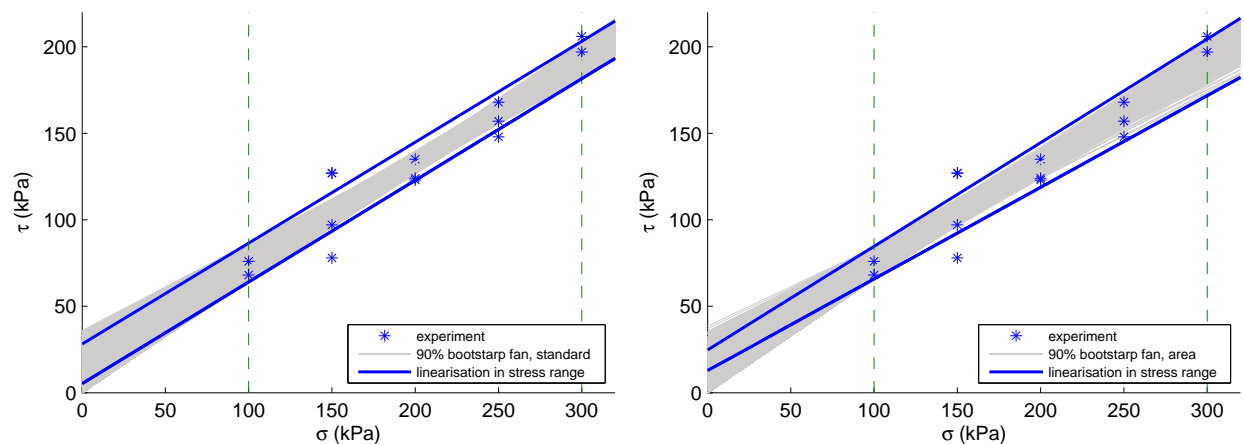

Figure 9 Bootstrap method with positivity constraint: remaining $90 \%$ fan of all realisations and linearisation in stress range, method standard vs. method area. The stress range for the geometrical enveloping is depicted by vertical dashed lines.

The results of all methods are summarised in Table 3. For comparison we included the estimation with the Bayesian approach.

As mentioned in Subsection 3.1, the numerical error in bootstrap resampling is of the order $1 / \sqrt{M}$. The scatter in the case of using method standard is smaller than in the case of using method area. In this example with 1000 realisations the resulting friction angles may vary by approximately 1 degree and the cohesions by $3 \mathrm{kPa}$. Clearly, increasing $M$ leads to smaller scatter; generally, $M$ should be chosen so that the numerical error remains in an acceptable range from the point of view of the intended application. 
Table 3 Limits of shear parameters estimated with various methods: $B S$ bootstrap, $n c$ no constraint, $p c$ positivity constraint, $n r$ negative rejected, area minimum area below shear strength line, standard fitted bivariate normal distribution of shear parameters.

\begin{tabular}{lcccr}
\hline Approach & $c_{5 \%}$ & $c_{95 \%}$ & $\varphi_{5 \%}$ & $\varphi_{95 \%}$ \\
\hline Classical regression & 0.0 & 24.2 & 31.0 & 31.3 \\
BS, nc, 0.9 area & 4.2 & 25.0 & 29.4 & 31.0 \\
BS, nc, 0.9 standard & -5.1 & 29.6 & 31.9 & 30.1 \\
BS, pc, 0.9 area & 11.7 & 25.1 & 28.3 & 31.0 \\
BS, pc, 0.9 standard & 5.3 & 28.3 & 30.5 & 30.3 \\
BS, nr, 0.9 area & 12.8 & 26.5 & 28.1 & 30.8 \\
BS, nr, 0.9 standard & 5.9 & 28.8 & 30.2 & 30.1 \\
Bayesian & 5.5 & 18.5 & 31.1 & 31.2 \\
\hline
\end{tabular}

\subsection{Bayesian approach}

The first series of the ring shear tests in Table 2 comprises $N=4$ experiments. Standard linear regression yields $c=13 \mathrm{kPa}$ and $\varphi=33^{\circ}$. Assessing characteristic shear parameters for this data set is possible with the Bayesian approach. We used the settings proposed in Section 3.3, that is, we fixed the regression variance $s_{\varepsilon}^{2}$ to the value given by Formula (8) with $\mathrm{SS}_{\mathrm{E}}$ from the standard linear regression. The prior distributions were taken to be uniform on the interval $[0,100](\mathrm{kPa})$ for the cohesion $c$ and on the interval $[0,1]$ for the friction coefficient $\nu=\tan \varphi$. The result is shown in Figure 10, with the confidence and linearised confidence limits for the shear strength lines on the left and the underlying posterior $90 \%$ confidence region for $c$ and $\nu=\tan \varphi$ on the right.
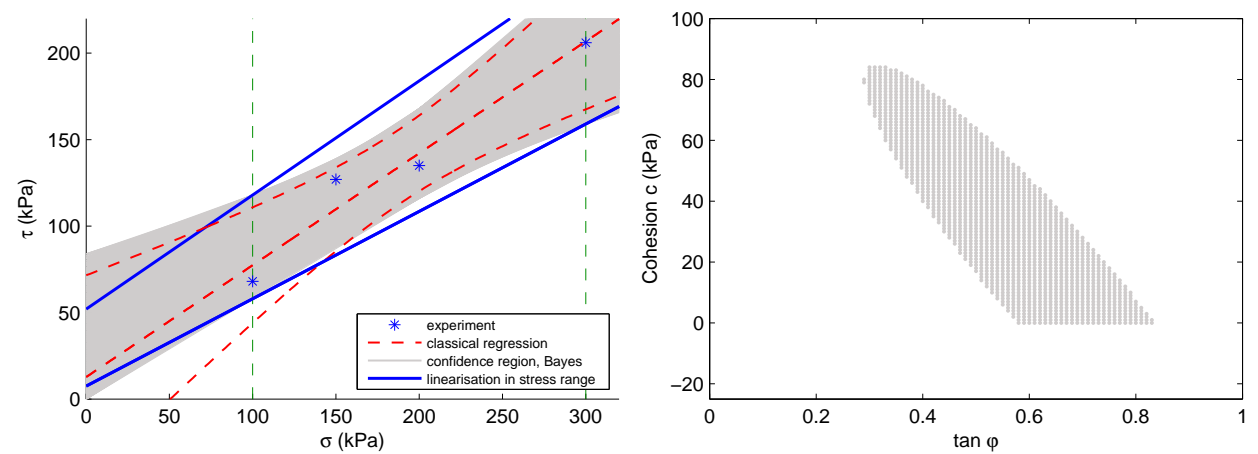

Figure 10 Result of the Bayesian approach with the first series in Table 2: regression (left), $90 \%$ confidence region (right). The stress range for the geometrical enveloping is depicted by vertical dashed lines.

Linearisation of the standard confidence limits would give a negative $c_{5 \%}$ for this example, whereas our proposed Bayesian approach yields a reasonable positive value, see Table 4 . Setting the minimum of the stress range (vertical dashed lines in Fig. 10-left) to zero would give $c_{5 \%}=0$. 
Table 4 Limits of shear parameters of the first series in Table 2 estimated with two methods.

\begin{tabular}{lcccr}
\hline Approach & $c_{5 \%}$ & $c_{95 \%}$ & $\varphi_{5 \%}$ & $\varphi_{95 \%}$ \\
\hline Classical regression & -17.8 & 43.2 & 31.7 & 34.1 \\
Bayesian & 6.0 & 45.0 & 28.4 & 33.4 \\
\hline
\end{tabular}

\section{Conclusion}

This article addresses the issue of determining characteristic soil parameters in geotechnical engineering. Eurocode 7 requires the provision of $5 \%$ and $95 \%$ confidence limits. We propose two methods for credibly determining such limits from direct shear tests, incorporating the constraint that the cohesion must be nonnegative and handling non-normality of distributions. The first method is applicable for sufficiently large sample sizes and employs bootstrap resampling to approximate the joint distributions of the cohesion and the friction angle. The second method can be used with small samples as well and is based on a Bayesian approach. Both methods require tools from computational statistics and are computer based.

The proposed methods should provide the geotechnician with a working procedure for arriving at the required characteristic values, using all available information in a systematic and transparent way.

\section{References}

Bauduin, Ch. (2002) 'Ermittlung charakteristischer Werte', in: Smoltczyk, U. (ed.): Grundbau-Taschenbuch, Teil 1. 6. Auflage, Ernst\&Sohn, Berlin, pp. 17-48.

CEN (2004), Eurocode 7: Geotechnical design - Part 1: General rules. European Committee for Standardization, Brussels.

Fellin, W., Berghamer, S. and Renk, D. (2009) 'Konfidenzgrenzen für die Scherfestigkeit als Grundlage zur Festlegung charakteristischer Scherparameter'. Geotechnik, Vol. 32 , pp. 30-36.

Gelman, A., Carlin, J.B., Stern, H.S. and Rubin, D.B. (2000) Bayesian Data Analysis. Chapman \& Hall/CRC, Boca Raton.

Kreinovich, V., Xiang, G. and Oberguggenberger, M. (2010) 'How to Define a Confidence Set for Functions: A New Justification of the Area Method'. Preprint.

Kruse, B. (2003) 'Status quo bei der Festlegung charakteristischer Werte von Bodenkenngrößen', in: Ziegler, M. (ed.): Beiträge zum Workshop Stochastische Prozesse in der Geotechnik. Schriftenreihe Geotechnik im Bauwesen, RWTH Aachen, pp. $43-53$.

Martz, H.F. and Waller, R.A. (2001) Bayesian Reliability Analysis. John Wiley \& Sons Ltd., Chichester.

Montgomery, D.C, Peck, E.A. and Vining, G.G. (1982) Introduction to Linear Regression Analysis. Third Ed., John Wiley \& Sons, New York.

Orr, T. (2000) 'Selection of characteristic values and partial factors in geotechnical designs to Eurocode 7'. Computers and Geotechnics, Vol. 26, pp. 263-279. 
Schneider, H.R. (1999) 'Definition and determination of characteristic soil properties', in: Proceedings of the Fourteenth International Conference on Soil Mechanics and Foundation Engineering, ICSMFE 14, Hamburg 199\%. A.A. Balkema, Rotterdam, Vol. 4, pp. 2271-2274.

B. Schuppener (1999), 'Die Festlegung charakteristischer Bodenkennwerte - Empfehlungen des Eurocodes 7 Teil 1 und die Ergebnisse einer Umfrage'. Geotechnik, Sonderheft, pp. 32-35.

Shao, J.and Tu, D.-S. (1995) The Jackknife and Bootstrap. Springer-Verlag, New York.

Viertl, R. (2003) Einfürung in die Stochastik. Mit Elementen der Bayes-Statistik und der Analyse unscharfer Information. 3. Auflage, Springer-Verlag, Wien.

Watabe, Y., Tanaka, M. and Kikuchi, Y. (2009) 'Practical determination method for soil parameters adopted in the new performance based design code for port facilities in Japan'. Soils and Foundations, Vol. 49, 827-839. 